\title{
Amygdaloid and hippocampal function in short-term retention of a classically conditioned fear response
}

\author{
MELVIN L. GOLDSTEIN and WILLIAM L. STOLLER \\ Indiana University, Kokomo, Indiana 46901
}

\begin{abstract}
An experiment was performed to investigate the effects of bilateral lesions in the amygdala and dorsal hippocampus on the retention and reconditioning of a classically conditioned fear response. The results of the experiment showed that both the amygdaloid and dorsal hippocampal lesions produced temporary retention deficits that were followed by permanent recovery of function after reconditioning. It was concluded that these areas are not the loci of the memory trace for the classically conditioned fear response. Perhaps more enduring memory deficits would be produced by larger lesions placed in the ventral hippocampus.
\end{abstract}

Horvath (1963) reported retention deficits after amygdalectomy in cats trained on a complex shuttle avoidance task that did not contain a discriminable cue as a background CS. Thatcher and Kimble (1966) found that overtrained rats showed little or no retention deficits of an avoidance response after bilateral amygdalectomy, whereas animals given no overtraining showed a marked retention deficit.

Goldstein (1975) subsequently demonstrated that rats with bilateral lesions of the basolateral amygdaloid complex could learn the classically conditioned fear response if the shock and safe compartments were made discriminably different. When the amount of fear reduction in the test situation was low, there was a greater performance deficit. The performance of amygdalectomized rats could be improved by introducing a discriminative cue into the test situation. The amount of fear reduction in the test situation was, therefore, probably a more important variable than the amygdalectomy in contributing to performance of the classically conditioned fear response.

The integrity of the amygdaloid complex is probably not directly involved in the retention of classical fear conditioning and avoidance conditioning. The amygdaloid complex may be essential for the temporary motivational processes involved in fear conditioning and avoidance conditioning, whereas the hippocampus may be important in processes of longer duration, such as shortterm memory or retention (Uretsky \& McCleary, 1969).

This hypothesis may be tested by determining whether permanent retention deficits can be demonstrated in hippocampal-lesioned rats under the conditions most conducive to maximal performance of the classically conditioned fear response (i.e., with the addition of the discriminative cue). Demonstration of permanent retention deficits in such preparations would suggest that the hippocampus rather than the amygdaloid complex is the significant structure in the retention of a classically conditioned fear response.

The main purpose of the present experiment was to determine the relative roles of the amygdaloid complex and the hippocampus in the short-term retention of a classically conditioned fear response. The test response used is simple, in the sense that the shock and safe compartments are discriminably different (i.e., the animals are run from a white shock compartment to a black safe compartment). An additional purpose of the experiment was to determine whether, if there is a retention deficit after placement of amygdaloid or hippocampal lesions, retraining can restore the original function. If a permanent retention deficit is found for the amygdaloid- or the hippocampal-lesioned rats, the specific area involved in the deficit may be a locus of the memory trace for the classically conditioned fear response.

\section{METHOD}

\section{Subjects}

The subjects were 30 naive male albino rats obtained from the Holtzman Company, Madison, Wisconsin. They were approximately 90 days old on arrival and 120 days old at the start of the experiment. They were maintained, ad lib, on Purina Rat Chow.

\section{Apparatus}

The apparatus was a black conditioning box divided by a guillotine-type door and a hurdle into two compartments of equal size. The shock compartment contained a grid floor and was backed with white cardboard. The safe compartment was painted flat black and contained a wooden floor. A buzzer and a light served as a compound CS. The UCS was an ac current conducted to the grid through a 250,000 -ohm series resistor. UCS intensity was $250 \mathrm{~V}$ ac, or $.83 \mathrm{~mA}$. Further details of the apparatus have been described earlier (Goldstein, 1977).

\section{Experimental Design}

The animals were trained on the classical fear conditioning test. They were given nine CS-UCS pairings at the medium UCS 
intensity in a white compartment (classical conditioning). They were then run from the white compartment to a black compartment for 20 test trials (instrumental hurdle-jump test trials). After a 10-min consolidation period, the animals received bilateral electrolytic lesions in either the basolateral amygdala or the dorsal hippocampus, or they were subjected to a sham operation. Two weeks later, they were given a retention test that consisted of 20 hurdle-jump trials. One week after the retention test, they were reconditioned. One week after the reconditioning session, they were given another retention test consisting of 20 hurdle-jump trials. There were 10 animals in each group.

\section{Procedure}

An experimental session lasted for $3 \mathrm{~h}$ and was divided into a classical conditioning and an instrumental test session. There were four experimental phases:

Phase 1. Exploration. The subject was placed into the grid compartment for a period of $10 \mathrm{~min}$. During this time, the subject explored both compartments. At the end of the 10-min exploration period, the guillotine door was lowered and the subject adapted to the grid compartment for $1 \mathrm{~h} 3 \mathrm{~min}$.

Phase 2. Classical conditioning. Each classical conditioning trial consisted of one presentation of CS and UCS while the subject was in the grid compartment, with the guillotine door closed. The interval between CS and UCS onset was $4 \mathrm{sec}$, UCS duration was $1 \mathrm{sec}$, and CS and UCS were terminated simultaneously. Successive CS-UCS presentations were separated by 3-min intervals. Subjects were given nine CS-UCS presentations.

The subjects were removed from the grid compartment 3 min after the start of the last conditioning trial. They were placed into the home cage in the animal room, where they were permitted to rest for $5 \mathrm{~min}$ with food and water available.

Phase 3. Exploration. A second 10-min exploration session in the conditioning box was given immediately afterward. A final 10 -min period in the home cage was given at the end of the second exploration session.

Phase 4. Instrumental test trials. Instrumental hurdle-jump conditioning sessions were started immediately after the last period in the home cage. The purpose of the hurdle-jump trials was to measure the strength of classical fear conditioning. Subjects jumped across a hurdle when the CS was turned on and the door separating the two compartments was opened. The CS was turned off as soon as the response had been made. Subjects were never given the UCS during this session and had been over the hurdle only during the 10 -min exploration session.

Each subject received 20 hurdle-jump acquisition trials during a $45-\mathrm{min}$ test period that began $28 \mathrm{~min}$ after the last classical conditioning trial. Hurdle-jump acquisition trials were separated by 2 -min intervals. Maximum CS duration permitted on any hurdle-jump test trial was $30 \mathrm{sec}$. If the subject had not crossed the hurdle by then, the experimenter lowered the door and, $10 \mathrm{sec}$ later, removed the subject from the grid compartment. Latencies of $30 \mathrm{sec}$ were recorded for such trails.

\section{Surgical and Histological Procedures}

The lesions were produced electrolytically with the aid of a Baltimore rat stereotaxic instrument. The coordinates used for the amygdaloid lesions were $2.5 \mathrm{~mm}$ posterior to bregma, $4.5 \mathrm{~mm}$ lateral to midline, and $8.0 \mathrm{~mm}$ below the dural tissue. Lesions were produced with a $2-\mathrm{mA}$ anodal current of $20 \mathrm{sec}$ duration. The stereotaxic coordinates used for the hippocampal lesions were $2.5 \mathrm{~mm}$ posterior to bregma, $2.0 \mathrm{~mm}$ lateral, and $3.3 \mathrm{~mm}$ down. A lesioning current of $5 \mathrm{~mA}$ was applied for $20 \mathrm{sec}$. For the sham-operated animals, a hole was drilled in the skull and the electrode was inserted to touch the dura, but no current was passed. All animals were maintained under sodium pentobarbital anesthesia (Nembutal, $60 \mathrm{mg} / \mathrm{kg}$ ip) during surgery.

At the conclusion of the experiment, the animals were sacrificed with sodium pentobarbital, and frozen coronal sec- tions of the brain were made and were examined microscopically. The location of the lesion was verified by comparing the sections with plates from the König and Klippel (1963) atlas.

\section{RESULTS}

The results of the experiment indicated that a retention deficit and permanent recovery of function occurred after placement of both basolateral amygdaloid and dorsal hippocampal lesions. There was, in addition, no significant difference between the retention deficits shown by dorsal hippocampal and basolateral amygdaloid animals $[t(18)=1.27]$. The latencies were transformed to reciprocals and plotted by blocks of five trials. The results of the experiment are shown in Figure 1.

A series of one-tailed $t$ tests was performed to test the significance of the performance deficits that occurred after the operations and the recovery of function that occurred with reconditioning. The drop in performance from the original conditioning after dorsal hippocampal lesions was significant at the .01 level of confidence $[t(18)=2.81]$. The drop in performance after basolateral amygdaloid lesions was significant at the .01 level of confidence $[t(18)=4.62]$. The drop in performance after the sham operation was not significant $(t=.36)$. The recovery of function after reconditioning for the dorsal hippocampal-lesioned group fell short of significance at the .05 level of confidence $[t(18)=1.55]$. For the amygdaloid-lesioned group, the recovery of function after reconditioning was significant at the .01 level of confidence $[t(18)=2.73]$. For the shamoperated group, the recovery of function was significant at the .05 level of confidence $[\mathrm{t}(18)=1.96]$. The recovery of function after reconditioning was

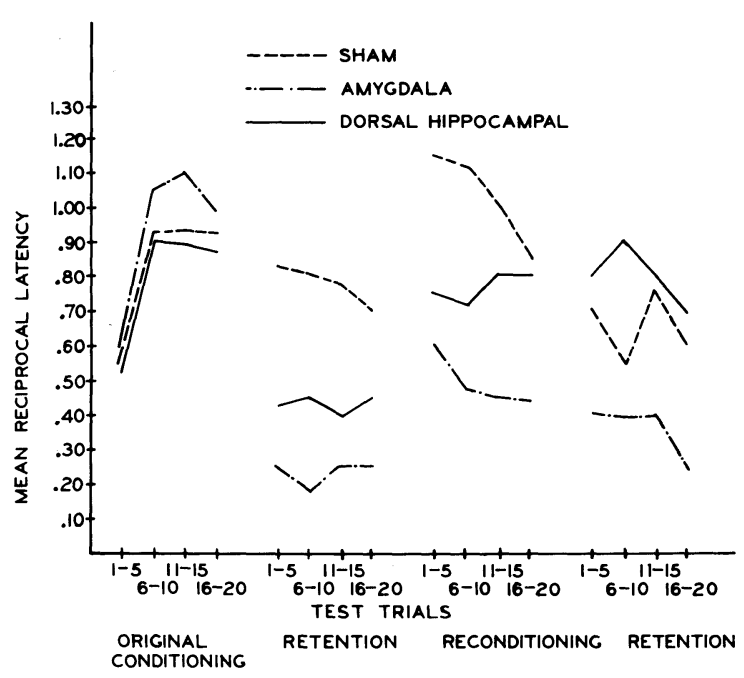

Figure 1. Effect of amygdaloid and dorsal hippocampal lesions on retention and reconditioning of the classically conditioned fear response. 
permanent for the groups, as indicated by the lack of retention deficit during the second retention session $(\mathrm{t}=0$ for the dorsal hippocampal group, $\mathrm{t}=.47$ for the basolateral amygdaloid group, $t=1.35$ for the shamoperated group).

The amygdaloid lesions typically included the entire basolateral region and most of the anterior amygdaloid area. Often the lesions extended into the corticomedial region, but the posterior one-fourth of the amygdaloid complex remained intact. Damage to the claustrum was commonly observed, and occasionally there was some damage to the putamen. The lesions in the dorsal hippocampus generally included all of the structure except the posterior one-fifth. The alveus and the corpus callosum were interrupted, and negligible damage was sustained by the fimbria. The lesions tended to extend dorsally into the cortex.

\section{DISCUSSION}

This experiment demonstrated that there were temporary performance deficits after both basolateral amygdaloid and dorsal hippocampal lesions. Recovery of function could be demonstrated after lesions in both areas. The recovery of memory was permanent for both dorsal hippocampal-and amygdaloidlesioned animals. The experiment did not discriminate between the temporary motivational factors and the longer duration associational factors, because the dorsal hippocampal animals showed relatively permanent recovery of performance. Permanent retention deficits could not be demonstrated for either the basolateral amygdaloid or the dorsal hippocampal animals.

Additional research is required to determine whether larger lesions in either the basolateral amygdaloid complex or the ventral hippocampus will produce lasting memory deficits. The experiment, therefore, yielded no positive evidence as to the locus of the memory trace for classical fear conditioning. Another possibility is that some other area of the brain, besides the amygdaloid complex or the dorsal hippocampus, may be responsible for memory for a traumatic event. The results of the experiment suggest that the amygdaloid complex and the dorsal hippocampus have similar or overlapping functions in the retention of a classically conditioned fear response.

Finally, the experiment looked at only limited areas of the amygdaloid complex and the dorsal hippocampus. These are relatively large and complex structures, and we may not have ablated the exact parts of these areas that are involved in the memory function. Medium-sized lesions were made in both areas. Perhaps larger lesions in the ventral hippocampus would produce more enduring memory deficits.

\section{REFERENCES}

Goldstein, M. L. Amygdalectomized rats can learn the classically conditioned fear response: A preliminary report. Bulletin of the Psychonomic Society, 1975, 6, 613-614.

Goldstein, M. L. Acquired drive strength as a joint function of UCS intensity and number of CS-UCS pairings. Journal of General Psychology, 1977, 96, 5-61.

HoRvath, F. E. Effects of basolateral amygdalectomy on three types of avoidance behavior in cats. Journal of Comparative and Physiological Psychology, 1963, 56, 380-389.

KöNIG, J. F. R., \& KLIPPEL, R. A. The rat brain: A stereotaxic atlas. Baltimore, Md: Williams \& Wilkins, 1963.

Thatcher, R. W., \& Kimble, D. P. Effect of amygdaloid lesions on retention of an avoidance response in overtrained and nonovertrained rats. Psychonomic Science, 1966, 6, 9-10.

URETSKY, E., \& MCCleary, R. A. Effect of hippocampal isolation on retention. Journal of Comparative and Physiological Psychology, 1969, 68, 1-8.

(Received for publication November 5, 1981.) 